\title{
Human Factor Based Failures in Technology Forecasting
}

Norbert Pałka ${ }^{a}$, Marcin Kowalski ${ }^{a}$, Tadeusz Szczurek ${ }^{a}$, Elżbieta Czerwińska ${ }^{a}$, Michał Walczakowski ${ }^{a}$, Artur Grudzien ${ }^{a}$,Alessandro Zanasi ${ }^{b}$, Graziano Giorgi ${ }^{b}$, Giulia Venturi ${ }^{b}$, Giuseppe Vella ${ }^{c}$, Piero Scrima ${ }^{c}$, Edward Hobbs ${ }^{d}$

a Military University of Technology, Urbanowicza Str. 2, 00-908 Warsaw, Poland http://www.wat.edu.pl/

b Zanasi \& Partners, Via Giardini, 4541124 Modena, Italy, https://www.zanasi-alessandro.eu/

${ }^{c}$ Engineering Ingegneria Informatica S.p.A., Piazzale dell'Agricoltura 24, 00144 - Roma, Italy, https://www.eng.it/

${ }^{d}$ Hawk Associates LTD, 66 Prescot Street, London E1 8NN, United Kingdom, https://www.hawkassociates/td.co.uk/

\section{A BSTRACT}

The subject of this paper is the technology forecasting failures. The topic is investigated by focusing on the human-related factors and cognitive pitfalls affecting human reasoning, based on a literature review. These factors are described and framed in the perspective of the seminal work by Kahneman. Four technology foresight errors made in the past by experts in the field are selected as representative case studies and analysed in terms of cognitive issues. Approaches to the problem are reported, considering the studies carried out in previous ECfunded project LEILA and RECOBIA. Finally, the outcomes of a questionnaire proposed within the EC-funded project PYTHIA are reported, outlining that

$\triangle$ Corresponding Author: Tel.: +48 261839936 Fax: +48 2266689 50; E-mail: norbert.palka@wat.edu.pl 
technology forecasting failure is still a prevailing problem and improvements are needed in terms of forecasting strategies and training for reducing cognitive pitfalls, highlighting the strategic importance of the afore-mentioned projects.

ARTICLE INFO

ReCEIVED: 01 Sep 2019

REVISED: 10 Nov 2019

ACCEPTED: 30 Dec 2019

ONLINE: 20 MAY 2020
KEYWORDS

cognitive biases, human factors, technology forecasting

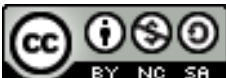

Creative Commons BY-NC-SA 4.0

\section{INTRODUCTION}

In the modern world, where the rapid development of new technologies and the implementation of new discoveries lead to a continuously increased complication of new ventures in many fields, the vision of the final goal is important, but possible failures must also be taken into account. Success is not only related to brilliant solutions but also to the capability of limiting the risk of failures and dead ends. This is, for example, the case of companies all around the world that are well aware of potential losses when their idea or product does not meet the customers' expectations or desires.

From a technical point of view, any potential failure connected to the development of new technology can be minimized by simulation, rigorous quality control, extended testing in the first stages of technology life cycle etc. But upstream of this, assumptions underlie the decisions made about a certain technology when it is just an idea or is in its first development stages. Such assumptions are based on predictions and judgements carried out by people, and may, therefore, be affected by deficiencies or pitfalls embedded in the human mind.

These cognitive pitfalls are investigated in section 2 of this paper, where a description of the most common human factor-related causes of errors during foresight activities, and human reasoning in general, is presented and framed in the theory of two reasoning systems by Kahneman [1]. Section 3 summarises the findings of two previous EC-funded projects LEILA [2] and RECOBIA [3], specifically dedicated to the mitigation of cognitive issues affecting decision-making in conditions of uncertainty, in particular within intelligence analysis. Section 4 aims at analysing four selected forecasting failures taken as representative case studies, attempting to understand the cognitive reasons behind them. Finally, the results of an online questionnaire arranged to collect experience of various domain experts concerning technology forecasting errors, are presented in Section 5, as part of the findings of the EC-funded project PYTHIA [4]. 


\section{HUMAN FACTORS IN FORECASTING}

A literature review on human factors-related errors during reasoning and forecasting highlighted the existence of voluminous material on the topic [5-30]. The most common cognitive errors are reported to be the following: representativeness, narrative fallacy, denial, the law of small numbers, availability bias, probability neglect, and anchor effect.

Representativeness is a simplified method of reasoning referring to the tendency to classify or judge based on partial similarities to what we already know, that is a typical, characteristic, representative, stereotypical image. When the human mind is requested to develop probability estimates, a common phenomenon is to base the estimate on what we already know, without taking into account the quality of the information. In some cases, this can lead to fast and correct ratings, but in other cases stereotypes give erroneous results [5-6].

The narrative fallacy refers to the illusion that one has comprehended the past factors that create the future, and hence he/she can predict or even control the future, or in other terms that stories from the past create our perception of the world and visions of the future [7]. Two factors contribute to the narrative fallacy. First, limitations arise from the possible lack of information or excessive complexity of information (epistemic uncertainty). Second, unpredictable interactions between phenomena do not allow to determine the degree of correctness of a system status forecast, due to the randomness of events, behaviours and their effects (aleatoric uncertainty). "Randomness is the lack of pattern or predictability of events" [8]. A random sequence of events is characterized by the fact that there is no logical order and is not compatible with the pattern or combination that people understand. This introduces the problem of predicting whether a given project or technical solution will be accepted by the target receiving group, or if a tendency will be maintained [9-10].

Denial is a defence mechanism (announced and studied by psychoanalyst Sigmund Freud [11]), usually manifesting itself in a scenario in which someone faces facts or information that are too uncomfortable for him or her to accept, and that are therefore refused despite evidence [12-13].

The law of small numbers is kind of reasoning bias (or error) which takes place when attributes or properties of a considered phenomenon are determined from a small number of observations or data points, ignoring that statistically only a large number of observations provides reliable result [14-15].

The availability bias is a kind of mental shortcut responsible for the fact that the human mind considers more significant something that can be easily retrieved from the memory, concerning other issues which are not as readily recovered. Events that particularly attracted attention, unpleasant events, and personal experiences that are easily recovered from memory, are weighted as more significant when making decisions and judgements. This is, for example, the case when each member of a working team feels that he/she works harder than others, and others 
are not grateful enough for their contributions: indeed, the majority of people thinks that their contribution is above average [16-17].

Probability neglect is the tendency to ignore a small risk or give it too much rank, distorting reality on the wave of the common spread and emotional intensity of the information available, thus focusing too much on events with very low probability and neglect highly probable events [18-19].

The anchoring effect is the tendency to stick too much to the first piece of information (anchor) that comes to mind when estimating an unknown value or entity. The human tendency is to proceed with subsequent adjustments of the original estimate, mentally moving away from the anchor. This may lead to big errors if the anchor was not carefully chosen. Anchoring also occurs as a suggestionbased effect [20-21].

All the afore-mentioned human factors-related errors are referred to as "cognitive biases" and are quite common during reasoning and forecasting. Daniel Kahneman established a cognitive biases theory that these common errors arise from simple but efficient rules which are often used to form judgements and make decisions and are called "heuristics". In his book "Thinking, Fast and Slow" [1] D. Kahneman describes two systems of thinking that directly translate into our decisions. System 1 represents a quick, emotional and intuitive way of thinking, which works automatically, usually with little effort and little sense of voluntary control. Slower operation, more deliberative and more logical thinking are the main features of system 2, which primarily focuses on mental effort.

Careless impressions and feelings of system 1 are the main sources of clear choices for system 2 . System 1 constantly creates suggestions - feelings, intentions, impressions, intuitions etc., which can be approved by system 2 and then turn into beliefs and voluntary activities. Usually, everything runs smoothly, and system 2 accepts an idea of system 1 . Indeed, people's first impression is correct in most cases. System 2 is activated for more detailed analysing of problems only when System 1 encounters difficulties and cannot propose a simple answer.

This arrangement of work between both systems is very productive and yields relatively high performance at low effort in every-day life because System 1 provides a fast and quite good model of situations, based on similar circumstances to previous ones. As a drawback, System 1 is prone to biases and features some systematic errors. Moreover, it goes shortcuts and has a small concept of logic and statistics.

Human beings usually believe that they are using logical system 2 and their choices are based on deep analysis, but many every-day decisions are usually made using system 1, following the very general principle of human action based on the "law of the least effort". System 1 provides fast and simple solutions based on feelings, impressions and intuitions, with low mental effort.

This is with no doubt very useful in every-day life but can lead to massive errors when it comes to providing estimations and forecasts. Indeed, the lack of awareness of this aspect is the key point when dealing with cognitive biases 
affecting decision-making and judgement, and even when awareness on this point is achieved, conscious limitation of the use of System 1 is still typically difficult to avoid, because it works automatically and has no stop button.

Nonetheless, strategies have been developed to mitigate cognitive biases and educate the human mind to consciously switch from System 1 to System 2 when delivering estimates, making decisions or giving judgements.

\section{OUtCOMES OF RELATED EC-FUndED PROJECTS}

In the period from February 2012 to January 2015, the European Project with acronym LEILA (Law Enforcement Intelligence Learning Applications) [2] was implemented. LEILA combined a psycho-sociological and cognitive factors-related approach to the problem of decision-making in conditions of uncertainty. The project provided new learning methods for intelligence analysis in support of law enforcement agencies. The main goal was to mitigate the causes of previous failures of intelligence analysis methods, specifically focusing on cognitive biases responsible for making incorrect decisions due to the lack of information or the availability of incomplete or non-reliable information.

The project took into consideration a questionnaire conducted by Robert Jervis [22], which found that intelligence failures can be reduced by ensuring continuous education of intelligence analysts. However, education without training is not enough, and to this regard, Heuer [23] published a study focusing on the necessity to provide training on cognitive bias, recognised as one of the main pitfalls that affect the reasoning aspects of the intelligence process.

Heuer suggested keeping the training program close to the real-world application through the use of simulations, games and other interactive teaching and experimental devices. The task was to deal with prejudices, fill the gap between human errors and logic, and fulfil user requirements and educational needs to improve the training of intelligence analysts.

Therefore, the LEILA project investigated how to take into account cognitive and decision biases when designing serious games dedicated to intelligence analysis. LEILA examined the links between the five phases of the intelligence process, i.e. planning and guiding, collecting, processing, analysis, and dissemination. It was found that specific cognitive errors affected analysts the most:

a) Confirmation bias, that may compromise the objectivity of analysts and disrupt the organization's decision-making process by neglecting conflicting evidence and assessments that do not cover the whole range of evidence [24];

b) Representativeness, giving the characteristics of a collective to people;

c) The availability heuristic, giving more probability to what is more available in our memory, what has happened to us or loved ones [25];

d) Anchoring, responsible for making decisions based too much on the tips given by the interlocutor, being subject to suggestions. 
Apart from LEILA, another EC-funded project was developed on the topic of cognitive biases: RECOBIA (REduction of COgnitive Biases in Intelligence Analysis [3]), lasted from 2012 to 2015, also investigated the impact of cognitive biases on the intelligence analysis and researched successful mitigation strategies to reduce them, taking into account the requirements and needs of end-users, gathered through meetings, workshops and interviews.

As a final result, a methodology was developed for the set-up of cognitive biasmitigation training programs, based on three steps:

1) to raise awareness of the course participants that cognitive biases pose a challenge to intelligence officers and that it is in the interest of every individual intelligence officer to know about them and know about strategies on how to mitigate them;

2) to introduce the participants into the underlying mechanisms of the cognitive biases, why they occur and why they are unavoidable, unconscious and involuntary;

3) to introduce the participant to the mitigation strategies; in the underlying mechanisms of mitigation strategies, why and how they work.

Also, the project developed a detailed risk analysis report on cognitive biases in the intelligence process and researched the best practices to reduce the negative impact of cognitive biases in the domains of software tools, training of analysts and organisational recommendations.

\section{HISTORICAL EXAMPLES OF TECHNOLOGY FORECASTING FAILURES}

The history of humanity provides many examples of human errors committed to forecasting the development of technology. This section presents an overview of four selected forecasting failures, based on literature, together with their analysis from the perspective of cognitive biases.

1) Dr. Lee De Forest was one of the American most famous scientists of his age, often referred to as "Father of electronics". However, despite his great scientific experience, he always rejected the idea of space travels. In 1952 he claimed that "spaceships to the moon or Mars" would have been technically impossible and that "mortals must live and die on Earth or within its atmosphere!". De Forest firmly maintained his beliefs even when the first space missions were approaching. In 1957 he declared to the Lewiston Morning Tribune that space flight by the means of rockets constituted "a wild dream worthy of Jules Verne". Furthermore, he also stated that that kind of voyage would have never occurred, regardless of all future advances [26]. In 1961 Yuri Gagarin completed an orbit of the Earth on a spacecraft and one year later Neil Armstrong and two others were the first men to step on the Moon.

This specific case study regards the fact that Lee De Forest believed space travels to be impossible and refused to change his mind, in principle. Scientists should always bear in mind that science describes the world through models and theories which can change and even be revolutionized by new discoveries as the 
technology evolves and more empirical results become available. Thus, this is an evident example of denial.

2) Sir Ernest Rutherford and his team were responsible for the discovery of the atomic in experiments between 1908 and 1913. This and other results earned him the title of "father of the nuclear physics" and a Nobel Prize in Chemistry in 1908. On September 1933, he stated in an interview on the New York Herald Tribune that there would have been no chance to produce energy breaking down atoms in laboratories. "Anyone who expects a source of power from the transformation of these atoms is talking moonshine" was a declaration that remained in the history of science [27]. Leo Szilard, a Hungarian-American physicist that was working on that specific problem during those days, read about Rutherford's declaration and was quite irritated by the great physicist's categorical statement. However, Rutherford was not alone in his negative opinion. Einstein compared the feasibility of transforming matter into energy to "shooting birds in the dark in a country where there are only a few birds [28]." Szilard, though, remained undeterred and a few months later proposed the idea of a nuclear chain reaction, turning things around. Without a chain reaction, Rutherford was effectively correct but Szilard's idea opened the way to nuclear fission and made the first nuclear reactor possible.

This specific case study regards the fact that Ernest Rutherford doubted that atomic manipulation would provide a viable source of energy, as indicated in a 1933 citation. The citation is extracted from an interview delivered to the New York Herald Tribune, a quite prestigious newspaper, reasonably read by a vast public, so that Rutherford's statement can be thought of having reached lots of people, including other scientific researchers, thus having impact also on the research orientation at that date. Connected to the previous forecast, also Einstein's opinion on the topic is reported, corroborating Rutherford's point of view.

These two forecasts, delivered by prominent world-famous vanguard physicists, were certainly due to an objective lack of knowledge at the time (here, a combination of epistemic and aleatory uncertainty), but they were also boosted by cognitive biases since scientists should always bear in mind that science describes the world through models and theories which can change and even be revolutionized by new discoveries as the technology evolves and more empirical results become available. In this case, to the contrary, Rutherford and Einstein denied the possibility of atomic power due to denial, availability bias and narrative fallacy.

3) Biosphere 2 was a project in which it was expected that an artificial biosphere that reproduced the terrestrial ecosystem in a small way would autonomously be regulated and made self-sufficient. It was a huge structure containing a sample of all ecosystems, capable of supporting a human population of "biospherians" indefinitely. Four men and four women began their isolation in September 1991. They would have been locked in Biosphere-2 for 2 years. Within a few weeks from the beginning of isolation, the oxygen level in the atmosphere had dropped from $21 \%$ to $14 \%$ [29], roughly as in the rarefied air on a $4000-$ meter mountain, just 
enough to keep the 8 members of the crew in health. At the same time, the $\mathrm{CO} 2$ level had increased incredibly, to the point of being close to the safety limit. All internal attempts to keep the situation under control aggravated the conditions of the structure. Nineteen of the twenty-five species of vertebrates, including all the fish, became extinct, as did all the pollinating insects, condemning most of the plants not to produce seeds. Most insects died quickly, except for cockroaches and ants. The artificial sea became acid and the corals began to die.

This case study regards the danger of setting up large-scale, expensive and highexpectation research projects without first checking the fundamental scientific assumptions at the base of the research. The project management and scientific management under-estimated the complexity of the Earth's natural biosphere, which is calibrated to cover an entire planet and not a small structure. Scientists failed in assessing the (very low) scalability of Nature: this is an example of anchoring bias.

4) The story of Digital Equipment Corp. (DEC) was one of a dramatic rise and fall, due to wrong predictions such as the one on the personal computer. DEC was an entrepreneurial computer company founded in 1957 that grew to \$ 14 billion in sales and employed an estimated 130,000 people worldwide at one point. In the 77 was quietly leader on the market with $41 \%$ of minicomputer sales in the world. A lot of minicomputer startup companies were founded in the Massachusetts region along route 128 during the 1950s as the Wang Laboratories in 1951 created by An Wang, an employee of Harvard Computational Lab. The minicomputer industry created work for about 500000 people in the region. But in the eighties came a threat and the strong rise of the Silicon Valley, where companies that were experimenting with new markets and new technologies, were growing. The era of the personal computer was coming. In DEC and the district of Massachusetts disapproved this trend and Ken Olsen (DEC co-founder) used to say phrases like "There was no reason for anybody to have a computer in his home". Within 10 years his multi-billion dollar mini-computer company would have no longer existed [30].

This case study regards the so-called CEO "bubble" or "disease", that is when a leader is walled in by self-importance and isolated from needed information. Kenneth Olsen based his marketing strategy and its productive chain orientation on a wrong assumption, forecasting that people would never need to have a computer in their home. Again, this is an example of anchoring bias.

\section{QUESTIONNAIRE RESULTS}

In this section, the analysis of the results of an online questionnaire are presented. The questionnaire was proposed within the work of the EC-funded project PYTHIA [4], which aims at developing a comprehensive and exhaustive methodology for the strategic technology foresight, intended as an intelligence activity. The questionnaire was set-up to directly collect the experience of PYTHIA's stakeholders, practitioners and domain experts concerning technology forecasting errors. The research was carried out from July to August 2018, and a total of 9 
responses were collected. This may appear a low number for a statistical approach, but still, the questionnaire provides relevant information, since the respondents were experts in the field.

The questionnaire was introduced by a general overview on the topic of technology failures, with the purpose to frame the problem. Then, the five main sections of the questionnaire were presented, each one divided into several specific sub-questions.

Section $A$ of the questionnaire focused on the identification of experts' technology areas and forecasting methodologies. The questionnaire's results outlined that forecasting is applied in several areas and to very different problems: from robotics to text mining, from mathematical algorithms to earth observation technologies, from cyber defence to laser and optoelectronics.

Section B of the questionnaire concerned forecast errors and forecast results. When asked "How it has been discovered that the prediction was wrong?", the respondents answered that they noticed the discrepancies only at the end of the work, and when comparing estimated data with real ones. Regarding the expression of the forecasts, some experts complained about how "blurry" the estimated scenarios were: this fact hindered decision-making and more realistic and defined views would have been preferred. Other respondents explained that predictions were incomplete and should have considered a longer time window.

Section C - "Details of errors" outlined that in the experts' experience two out of three predictions have been proved to be - at least partially - incorrect. The main reasons for these failures were reported to be inaccurate sources selection and validation, methodological errors, the fact that only the technical aspects were considered, cognitive errors (misinterpretation of data, improper assignment of causal relationships between data), biases such as confirmation bias, and too much trust in the IT software. It is worth noting that all experts stated that several errors happened due to unconscious issues.

Consequences of the prediction process errors were investigated in Section D of the questionnaire, and resulted to be quite serious: loss of strategic superiority, reputation damages and delayed development were mentioned.

Finally, section $\mathrm{E}$ revealed that some experts were unsure about how to remove cognitive errors from their forecasting process, underlying the need for dedicated and comprehensive recommendations and best practices to tackle these cognitive pitfalls.

\section{SUMMARY AND CONCLUSIONS}

In this paper, the subject of failures of the technology forecast was undertaken. The topic was firstly investigated from a general perspective, researching common errors in human reasoning, through a literature review. Then, an anthropologic explanation was proposed for these errors, based on the theories of Kahneman. Approaches to the problem were reported, considering the studies carried out in previous EC-funded project. After, the proper technology-related framework was 
explored, reporting notable examples of technologic foresight failures selected as representative case studies.

Finally, the outcomes of a questionnaire proposed within the EC-funded PYTHIA project were reported, specifically tailored for the technology foresight domain. Respondents outlined the fact that they had experienced technology-related forecasting failures, related both to strictly technical aspects and to human-related factors. Some of the reported failures could have been avoided by additional quality control, modelling and simulations, but several other failures happened due to unconscious issues. Regarding this, respondents stated that they were unsure about how to remove cognitive errors from the work process or at least reduce them.

In this frame, the findings of the LEILA and RECOBIA projects concerning education and training of analysts against cognitive biases appear particularly useful and may lead to improved forecast accuracy and reduced failure potential.

In addition to this, since the questionnaire's results highlighted that failures were encountered in a variety of technological fields (such as text mining, mathematical algorithms, lasers and unmanned vehicles and drones), the general and comprehensive methodology developed in PYTHIA appears to be exceptionally useful. It should be noted that the methodology, apart from the specific training and recommendations regarding the psychological strategies for the mitigation of cognitive biases, also comprehends the descriptions of the available software tools and technological solutions that may support analysts in making sense of the available data, if used properly.

All the proposed strategies should be combined and taken into consideration to reduce errors and failures within technology forecasting, with the purpose to prevent serious consequences including loss of strategic superiority, reputation damages, delayed development of technology and lost chances for business. These issues will be considered as starting points for future works carried out by the PYTHIA consortium.

\section{ACKNOWLEDGEMENTS}

This article has been produced as a result of PYTHIA project. PYTHIA (Predictive methodologY for TecHnology Intelligence Analysis) has received funding from the European Union's Preparatory Action on Defence Research under grant agreement No 800893.

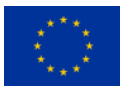




\section{DISCLAIMER}

The information contained in this document reflects only the author's view and the European Defence Agency is not responsible for any use that may be made of the information it contains

\section{REFERENCES}

[1] D. Kahneman, "Thinking, Fast and Slow", New York: Farrar, Straus and Giroux, 2011.

[2] http://leila.fvaweb.eu/

[3] https://www.recobia.eu/

[4] www.pythia-padr.eu

[5] Tversky, A., Kahneman, D., "Judgement under uncertainty: Heuristics and biases". Science. 185 (4157): 1124-1131, 1974, doi:10.1126/science.185.4157. 1124.

[6] Gilovich, Thomas; Savitsky, Kenneth (1996). "Like Goes with Like: The Role of Representativeness in Erroneous and Pseudo-Scientific Beliefs". Sceptical Inquirer. 20 (2): 34-40. doi:10.1017/СB09780511808098.036.

[7] A. Tversky, D. Kahneman, 1973 "Availability: A Heuristic for Judging Frequency and Probability. Cognitive Psychology", 5, 207-232, 1973.

[8] https://en.oxforddictionaries.com/definition/randomness

[9] Church, Alonzo, "On the Concept of Random Sequence". Bull. Amer. Math. Soc. 46 (2): 130-136, 1940. doi:10.1090/S0002-9904-1940-07154-X.

[10] Hazewinkel Michiel, "Random sequence", Encyclopedia of Mathematics, Springer Science+Business Media B.V. / Kluwer Academic Publishers, 2001, ISBN 978-1-55608-010-4.

[11] Sigmund Freud "The Neuro-Psychoses of Defence", 1894

[12] Niolon, Richard, "Defenses". psychpage.com, (April 8, 2011).

[13] Plutchik, R., Kellerman, H., \& Conte, H. R., "A structural theory of ego defences and emotions." In C. E. Izard (Ed.), Emotions in personality and psychopathology (pp. 229--257), 1979. New York: Plenum Press.

[14] Richard K. Guy, "The Strong Law of Small Numbers", American Mathematical Monthly. 95 (8): 697-712, 1988. doi:10.2307/2322249.

[15] Amos Tversky; Daniel Kahneman, "Belief in the law of small numbers". Psychological Bulletin. 76 (2): 105-110, 1971. doi:10.1037/h0031322

[16] Sjöberg, Lennart; Engelberg, Elisabeth, "Risk Perception and Movies: A Study of Availability as a Factor in Risk Perception". Risk Analysis. 30 (1): 95-106, 2010. doi:10.1111/j.1539-6924.2009.01335.x. 
[17] Kliger, Doron; Kudryavtsev, Andrey, "The Availability Heuristic and Investors' Reaction to Company-Specific Events". Journal of Behavioral Finance. 11 (1): 5065, 2010. doi:10.1080/15427561003591116. ISSN 1542-7560.

[18] Sunstein, Cass, "Probability Neglect: Emotions, Worst Cases, and Law", 2001. SSRN 292149.

[19] Sunstein, Cass, "Terrorism and Probability Neglect". Journal of Risk and Uncertainty. 26 (2): 121-136, 2003. doi:10.1023/A:1024111006336.

[20] Mussweiler, Thomas; Strack, Fritz, "Hypothesis-Consistent Testing and Semantic Priming in the Anchoring Paradigm: A Selective Accessibility Model". Journal of Experimental Social Psychology. 35 (2): 136-164, 1999.

[21] Furnham, Adrian; Boo, Hua Chu, "A literature review of the anchoring effect", The Journal of Socio-Economics. 40 (1): 35-42, 2011. doi:10.1016/j.socec. 2010.10.008.

[22] Robert Jervis, "Why intelligence fails", ISBN 0801447852, 2010.

[23] Heuer, R.J., "The Psychology of Intelligence Analysis", Washington DC: Center for the Study of Intelligence, Central Intelligence Agency, 1999. "

[24] Wason, P.C., "On the Failure to Eliminate Hypotheses in a Conceptual Task", Quarterly Journal of Experimental Psychology, 12 (3), 129-140, 1960.

[25] P. Pirolli, S. Card, "The Sensemaking Process and Leverage Points for Analyst Technology as Identified Through Cognitive Task Analysis", Proceedings of International Conference on Intelligence Analysis. Retrieved on 21/07/2014, 2005; from: http://vadl.cc.gatech.edu/documents/2 card-sensemaking.pdf

[26] "De Forest Says Space Travel Is Impossible", Lewiston Morning Tribune, February 25, 1957

[26] Howard Mingos, ed. "The Aircraft Year Book for 1935", New York: Aeronautical Chamber of Commerce of America, Inc., 1935. 248.

[27] "Rutherford cools atom energy hope". The New York Times, September 12, 1933.

[28] Brian Van DeMark, "Pandora's Keepers: Nine Men and the Atomic Bomb"

[29] Severinghaus, J.P., Wallace, S.B., William, F.D., MacCallum, T., Whalen, M., "Oxygen loss in biosphere 2", Eos 75, 33-37, 1994.

[30] "Creative Computing" magazine, April 1980. Edited by David H. Ahl. 